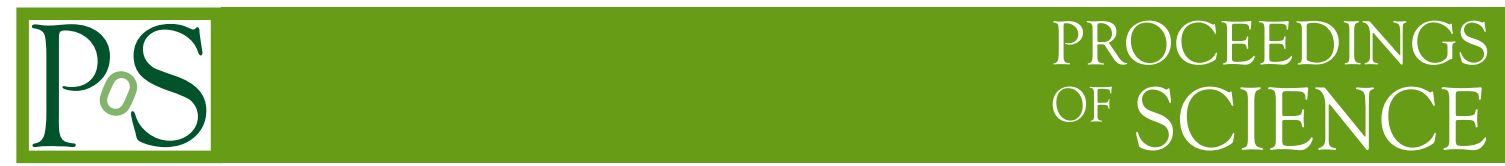

\title{
CU Virginis - A unique stellar pulsar?
}

\section{Barry Kellett*}

Rutherford Appleton Laboratory

E-mail: B.J.Kellett@rl.ac.uk

CU Virginis is one of the brightest radio emitting members of the magnetic chemically peculiar (MCP) class of stars and also one of the fastest rotating. We have now confirmed that CU Vir is unique among stellar radio sources in generating a persistent, highly collimated, beam of coherent, $100 \%$ right-hand polarised, radiation from one (at least) of its magnetic poles that sweeps across the sky in a wide emission cone that intercepts the Earth twice every time the star rotates. This makes the star strikingly similar to a pulsar. This similarity is further strengthened by the observation that the rotating period of the star is lengthening at a rapid rate due to a braking mechanism probably related to its very strong magnetic field (and possibly its oblique rotation configuration).

From Planets to Dark Energy: the Modern Radio Universe

October 1-5 2007

The University of Manchester, $U K$

\footnotetext{
*Speaker.
} 


\section{Background}

When simultaneous data on active stars from the radio and X-ray wavebands are compared a remarkable correlation seems to apply - ranging from solar flares to active dwarf and giant stars covering 8-10 orders of magnitude [1]. But why should the X-ray and radio fluxes correlate at all? Then, in 1998, the first resolved radio picture of a star was obtained [2]. This showed that the emission was coming from the polar regions of the star (as determined by the plane of the binary orbit). This is quite unlike the Sun. The final piece of the puzzle was found when the radio flare emission was compiled. For 15 of 17 radio flares from the star UV Ceti, the emission was found to be highly right-hand circularly polarized (RCP) upto $100 \%$ in many cases. (The two exceptions were $100 \%$ left-hand polarized, but very intense and short lived bursts [3].)

It was this last observation that suggested that the radio emission model assumed might be inapplicable. The model assumes that the emission is gyrosynchrotron produced by $\mathrm{GeV}$ electrons and a magnetic field. The X-rays only require $\mathrm{keV}$ electrons and it seems hard to believe that a star can simultaneous produce copious amounts of $\mathrm{keV}$ and $\mathrm{GeV}$ electrons at the same time. Could a new radio emission mechanism be found that would work with just keV electrons? And what about the $100 \%$ right-hand circular polarization? The answer seemed to be a coherent generation process, such as a cyclotron maser, but how was it excited/driven? The answer was to assume a relatively simple magnetic field geometry for the star, a toriodal dipole magnetic field.

This is actually exactly like the magnetic field of the Earth. The Earth is known to emit radio emission, and it emanates from the polar (or auroral) regions, and it is $100 \%$ RCP. In fact, the Earth's Auroral Kilometric Radiation (AKR) is remarkably similar to the radio flare emission of UV Ceti, except for the wavelength of the emission, $\mathrm{cm}$ for the star and $\mathrm{km}$ for the Earth. But this simply reflects the vastly different magnetic field strengths of the two sources.

\section{New Cyclotron Maser Mechanism}

AKR requires a low density cavity and an electron beam to enter this region. The electron beam is subjected to substantial adiabatic magnetic compression as it descends into the planetary magnetic dipole resulting in the conversion of axial momentum into rotational motion at the local electron cyclotron frequency. Theoretical analysis indicates that the electron beam will form a crescent or horse-shoe form of distribution function. Such a distribution is unstable to the emission of radiation at the cyclotron frequency in the $\mathrm{X}$-mode $[4,5]$. This new cyclotron maser mechanism can replace the well known loss-cone mechanism in many situations because it is much simpler to generate the new radiation, it simply requires a converging dipole magnetic field and an electron beam!

This new mechanism naturally explains why the radio emission is $100 \% \mathrm{RCP}$ and why it is from the auroral regions of the star, and also why the star does not require $\mathrm{GeV}$ electrons.

The mechanism works so well that it can be can and indeed has been tested in the laboratory, in an experiment that is $\sim 1.5$ meters long and generates cyclotron maser radiation in the $\mathrm{GHz}$ regime[6]. 


\section{New Cyclotron Star}

CU Virginis is a magnetic chemically peculiar star. This means it possesses a strong dipole magnetic field and we know this is offset from the rotational axis of the star. So, as the star rotates, our view of the magnetic field changes. That means that for each rotation of the star (about 12.5 hours) we pass through the magnetic equator twice and our line-of-sight also passes close to both magnetic poles. VLA observations in 1998 showed that CU Vir can produce highly polarized radio emission, in short "bursts". This strongly suggested to us that the star is generating cyclotron maser radiation via our new mechanism.

We have now observed the star with MERLIN and confirmed that CU Vir produces repeating, narrow, $100 \%$ right-hand circularly polarised (RCP) emission at $1.6 \mathrm{GHz}$. We believe that the two narrow emission peaks are the two sides of an open emission cone from just one of the magnetic poles of the star. Another possible application of the new radio emission mechanism is to explain the intense radio emission from quasar jets, in particular from blazars[7].

\section{References}

[1] Gudel, M., Schmitt, J.H.M.M., Benz, A. O. Microwave emission from X-ray bright solar-like stars: the F-G main sequence and beyond. Astronomy and Astrophysics 302, 775, 1995.

[2] Benz, A. O., Conway, J., Gudel, M. First VLBI image of a main-sequence star Astronomy and Astrophysics 331, 596, 1998.

[3] Kellett, B. J., Bingham, R., Cairns, R. A., Tsikoudi, V. Can late-type active stars be explained by a dipole magnetic trap? Monthly Notices of the Royal Astronomical Society 329, 102-108, 2002.

[4] Bingham, R., Cairns, R. A. Generation of auroral kilometric radiation by electron horseshoe distributions. Physics of Plasmas 7, 3089-3092, 2000.

[5] Bingham, R., Cairns, R. A., Kellett, B. J. Coherent cyclotron maser radiation from UV Ceti. Astronomy and Astrophysics 370, 1000-1003, 2001.

[6] Cairns, R. A., Speirs, D. C., Ronald, K., Vorgul, I., Kellett, B. J., Phelps, A. D. R., Bingham, R. A Cyclotron Maser Instability with Application to Space and Laboratory Plasmas. Physica Scripta Volume T 116, 23-26, 2005.

[7] Bingham, R., Kellett, B. J., Cairns, R. A., Tonge, J., Mendonça, J. T. Cyclotron Maser Radiation from Astrophysical Shocks. Astrophysical Journal 595, 279-284, 2003. 